\title{
Article
}

\section{Sub-clinical Detection of Left Ventricular Myocardial Dysfunction in Valvular Heart Diseases: A State-of-the-Art Review in a Speckle Tracking Echocardiography and Myocardial Performance}

Elkilany, Galaleldin Nagib, Allah, Sherif Baath, Lohana, Petras, Sozzi, Fabiola, Singh, Jaipaul, Khorshid, Mohamed, Singh, Ram B and Aiash, Hani

Available at http://clok.uclan.ac.uk/34189/

Elkilany, Galaleldin Nagib, Allah, Sherif Baath, Lohana, Petras, Sozzi, Fabiola, Singh, Jaipaul ORCID: 0000-0002-3200-3949, Khorshid, Mohamed, Singh, Ram $B$ and Aiash, Hani (2020) Sub-clinical Detection of Left Ventricular Myocardial Dysfunction in Valvular Heart Diseases: A State-of-the-Art Review in a Speckle Tracking Echocardiography and Myocardial Performance. Journal of Cardiology Research Review \& Reports, 1 (3). pp. 11-13.

It is advisable to refer to the publisher's version if you intend to cite from the work.

For more information about UCLan's research in this area go to http://www.uclan.ac.uk/researchgroups/ and search for <name of research Group>.

For information about Research generally at UCLan please go to http://www.uclan.ac.uk/research/

All outputs in CLoK are protected by Intellectual Property Rights law, including Copyright law. Copyright, IPR and Moral Rights for the works on this site are retained by the individual authors and/or other copyright owners. Terms and conditions for use of this material are defined in the policies page. 


\title{
Journal of Cardiology Research Review \& Reports
}

\section{Sub-clinical Detection of Left Ventricular Myocardial Dysfunction in Valvular Heart Diseases: A State-of-the-Art Review in a Speckle Tracking Echocardiography and Myocardial Performance}

\author{
Galaleldin Nagib Elkilany ${ }^{1,2 *}$, Sherif Baath Allah ${ }^{3}$, Petras Lohana ${ }^{3}$, Fabiola Sozzi ${ }^{4}$, Jaipaul Singh $^{5}$, Mohamed Khorshid ${ }^{6}$, Ram B Singh ${ }^{7}$ and $^{2}$ \\ Hani Aiash ${ }^{8}$
}

${ }^{1}$ Assistant Clinical Professor - SCU Gulf Medical University, Ajman, UAE

${ }^{2}$ Consultant Cardiologist at Elaj Medical Center, Burjeel Specialty Hospital and Kings College Hospital London, Dubai, UAE

${ }^{3}$ Associate Professor at RAK Medical and Health Science University and Masafi Hospital, UAE

${ }^{4}$ Policlinico University Hospital IRCCS, Milan, Italy

${ }^{5}$ School of Forensic and Applied Sciences, University of Central Lancashire, Preston, UK

${ }^{6} \mathrm{MBBCh}$, Faculty of Medicine, Zagazig University

${ }^{7}$ Halberg Hospital and Research Institute, Moradabad, India

\begin{abstract}
Purpose of the state-of-the-art review: Left ventricular (LV) global longitudinal strain (GLS) is recently recognized as a more sensitive measure of LV myocardial systolic function compared with LV ejection fraction (LVEF). In addition, left ventricular GLS , myocardial performance index (MPI) and maximum rate of $\mathrm{LV}$ pressure rise during isovolumetric contraction $\left(\mathrm{LV} \mathrm{dP} / \mathrm{dt}_{\max }\right.$ ) are more reproducible than traditional assessment of $\mathrm{LV}$ systolic function by two dimensional echocardiography (2DE) LVEF. These underutilized techniques can detect preclinical myocardial dysfunction in patients who are at risk of LV failure in valvular-induced heart disease . Current guidelines for diagnosis and treatment of valvular heart disease (VHD) include LVEF as one of the parameters to take into consideration in the clinical decision-making. However, a large body of evidence has shown that left ventricular GLS, MPI and LV dP/dtmax have been classically considered as a sensitive marker of LV contractility and inotropic state. In turn GLS and myocardial performance may be a better prognosticator than LVEF in aortic and mitral valve heart diseases. This timely state-of-the-art review, appraised the evidence and role of GLS, MPI and $\mathrm{dP} / \mathrm{dT}$ as clinical tools in patients with aortic and mitral valve disease.
\end{abstract}

Recent findings: Left ventricular GLS has been shown to be prognostic in low-flow, low-gradient severe aortic stenosis with preserved LVEF. The role of left ventricular GLS, Tei index (MPI) and maximum rate of $\mathrm{LV}$ pressure rise ( $\mathrm{LV} \mathrm{dP/dt}$ ) in patients with aortic regurgitation and mitral valve diseases (regurgitation and stenosis) is less well established.

\begin{abstract}
Summary: Echocardiography is considered the primary non-invasive imaging tool for valvular heart disease assessment and the cornerstone method in diagnosing and evaluating the morphology and severity of aortic and mitral valve diseases. Currently, diagnostic-cardiac catheterization is no more recommended except in very rare cases when echocardiographic image quality is suboptimal, non-diagnostic and when the results of $2 \mathrm{DE}$ are discrepant with clinical data. Once clinical decision-making is based on the $2 \mathrm{DE}$ and three dimensional echocardiographic in assessment of the severity of mitral and aortic valve diseases, it is crucial that standards should be adopted to maintain accuracy and consistency across echocardiographic laboratories. This illustrative review article assesses left ventricular systolic function (LVEF) employing two and/or three dimensional echocardiography in comparison to GLS, MPI and LV dP/dt ${ }_{\max }$ especially applied for aortic valve (AV) and mitral valve (MV) diseases. It is noteworthy that this document only provides echocardiographic standards rather than making recommendations for clinical management.

Conclusion: It is concluded that GLS, MPI and maximum rate of LV pressure rise during isovolumetric contraction (LV $\mathrm{dP} / \mathrm{dt}_{\text {max }}$ ) are recommended and more so, they should be increasingly used to identify subclinical LV myocardial dysfunction in patients with mitral and aortic valve heart diseases, to identify optimal timing for surgery and prognosticate outcomes after surgery.
\end{abstract}

\section{*Corresponding author}

Galaleldin Nagib Elkilany, Assistant Clinical Professor and Consultant of Cardiology at Gulf Medical University (GMU), UAE. E-mail: Galal. elkilany@gmail.com

Received: July 17, 2020; Accepted: July 22, 2020; Published: July 25, 2020 
Keywords: Valvular heart disease, echocardiography, left ventricle, myocardial function

\section{Introduction \\ Echocardiographic Techniques for Strain Assessment}

Tissue Doppler imaging (TDI) or speckle tracking echocardiography (STE) is used to measure myocardial deformation. TDI was the first echocardiographic technique for obtaining strain measurements. It has been well validated and has the highest temporal resolution among all deformation imaging modalities $[1,2]$. However, the necessity of additional image acquisitions and post-processing for a comprehensive LV analysis are major limitations for routine clinical application. The newer approach, based on tracking speckles from frame to frame in the regular gray scale image, enables a fast and user-friendly semi- or fullyautomatic strain analysis, which can improve its usefulness in clinical practice. However, STE depends on good image quality and geometry, which limits its applicability in patients with suboptimal echogenicity. Recently, algorithm-related inter vendor differences have been substantially reduced following efforts of the European Association of Cardiovascular Imaging, American Society of Echocardiography and Industry Task Force on strain standardization [2]. Current studies suggest that STE might identify asymptomatic patients with severe aortic stenosis who would benefit from earlier intervention than recommended in recent guidelines $[2,3]$.

The problems with image quality, frame rate, and inter vendor differences are even more pronounced in 3D STE compared with 2D STE [4-6].

\section{Assessing Cardiac Function in Valve Disease : Lv Ejection Fraction Versus Strain (Gls)}

LVEF is incorporated into current guideline-recommended treatment strategies for valve diseases, in order to perform interventions in asymptomatic patients with severe valvular dysfunction before developing irreversible LV damage [7]. Therefore, surgery is recommended in a symptomatic patient with severe mitral regurgitation (MR) with early signs of LV systolic dysfunction (LVEF $\leq 60 \%$ and/or LV end-systolic diameter $\geq 45$ $\mathrm{mm})$. Treatment is recommended at LVEF $<50 \%$ in patients with severe aortic regurgitation or aortic stenosis. However, assessment of systolic dysfunction in valvular heart disease is challenging. Furthermore, longitudinal strain has been shown to be a strong predictor of all- causes of mortality independent of aortic stenosis severity and EF or other known predictors [8].

LVEF represents only a relative volume change from enddiastole to end systole and does not account for the complexity of myocardial mechanics and varies in response to pre load and after load changes. This volume-based parameter (LVEF) has an important limitation in reflecting systolic function in altered loading (pressure and volume) conditions, which are permanently present in patients with valve diseases. LVEF is often in the "supernormal" range in aortic and mitral incompetence lesions and therefore a poor representative of systolic myocardial function $[9,10]$.

In summary, although GLS is of crucial importance in early detection of sub clinical LV myocardial dysfunction and predictive of all-cause mortality independent of aortic stenosis severity and ejection fraction. 2DE dimensions (end systole and end diastole LV dimensions) and systolic function indices (LVEF) and fractional shortening (FS) are still considered essential in determining the timing of surgery in aortic and mitral valve disease.
In mitral regurgitation, $\mathrm{LVEF}$ overestimates cardiac function as it reflects both the aortic forward stroke volume and regurgitant volume pumped into the low pressure left atrium. Consequently, LVEF can remain in a normal range for a long time and may mask subtle early reductions in contractility, despite intrinsic myocardial dysfunction [10].

In aortic valve (AV) stenosis (and pulmonary valve) lesions, present with concentric remodeling with increased wall thickness and reduced cavity diameter; hence LVEF can remain preserved despite reduced shortening of the myofibrils [10,11]. Therefore, LVEF can only detect LV systolic dysfunction in a relatively advanced stage of disease when a relevant myocardial contractile dysfunction has already developed. Myocardial deformation imaging, however, may be superior in detecting subtle dysfunction [9-11]. Longitudinal LV strain (GLS) is the most vulnerable component of LV mechanics. In an early stage of disease, impaired longitudinal deformation is often compensated by an augmented circumferential function, which keeps the LVEF within a normal range $[11,12]$. It has been shown that circumferential strain contributes more than twice as much to LV as longitudinal strain. Therefore, detection of early cardiac dysfunction in valve disease is one of the most promising indications of strain imaging [13-17].

\section{Myocardial Performance Index (Tei Index) and Maximum Rate of LV Pressure Rise ( $\mathrm{LV} \mathrm{dP/dt} \mathrm{max}_{\text {ax }}$ )}

In addition to GLS and LVEF; a large body of evidence is now emerging highlighting the accurate assessment of LV and right ventricular contractile functions are possible in advanced aortic and mitral valve diseases at subclinical stage. Myocardial performance index (Tei Index) $>0.42$ with increased B type natriuretic peptide (BNP) levels $>550 \mathrm{pg} / \mathrm{ml}$ and depression of maximum rate of LV pressure rise $\mathrm{LV}\left(\mathrm{dP} / \mathrm{dt}_{\max }\right)$ are better alternatives to LVEF in the presence of mitral and aortic valves regurgitation, for sub clinical detection of LV myocardial dysfunction [18-24,25].

Early studies showed that $\mathrm{dP} / \mathrm{dt}_{\text {max }}$ and MPI could Identify latent systolic dysfunction of LV and post-operative risk in patients with mitral and aortic valve diseases, even when LVEF was still preserved [18-23,25].

\section{The Crucial Role of Echocardiography and Novel Parameters of LV Function Assessment in a Special Sub Groups of Patients With Valvular Heart Diseases}

Aortic Valve Stenosis (AS): Discordance Between Velocity And Aortic Valve Area (AVA) : Low flow AS, paradoxical low flow AS, associated aortic valve regurgitation (AR), hypertrophic cardiomyopathies (HCM) and arterial Hypertension (HTN) ,all can interfere with proper assessment of AS severity.

The role of Dobutamine-Induced Stress chocardiography (DSC) for Evaluation of as Severity in the Setting of LV Dysfunction. The findings which are highly suggestive of severe stenosis is an increase of the mean gradient to $>30-40 \mathrm{~mm} \mathrm{Hg}$ or a jet velocity $\geq 4.0 \mathrm{~m} / \mathrm{s}$ provided that valve area does not exceeding $1.0 \mathrm{~cm} 2$ at any flow rate [26]. On the other hand, an increase in effective aortic valve area (AVA) to a final valve area $>1.0 \mathrm{~cm} 2$ suggests that stenosis is not critically severe [27]. Furthermore, increase in SV by $>20 \%$ from baseline suggests presence of contractile reserve , which has important clinical and therapeutic implication in low flow AS . Alternatively, absence of contractile reserve is a predictor of a high post-operative mortality and poor long-term outcome [28]. Another important application of DSE is an association of valvular AS with coronary artery disease (CAD) which can be 
seen in up to $50 \%$ in elderly AS patients. An improvement of contractility of either a kinetic-hypokinetic or dys-synergic myocardial segment or biphasic response during DSE is considered as a sign of viability even if the ischemic myocardium is in jeopardy.

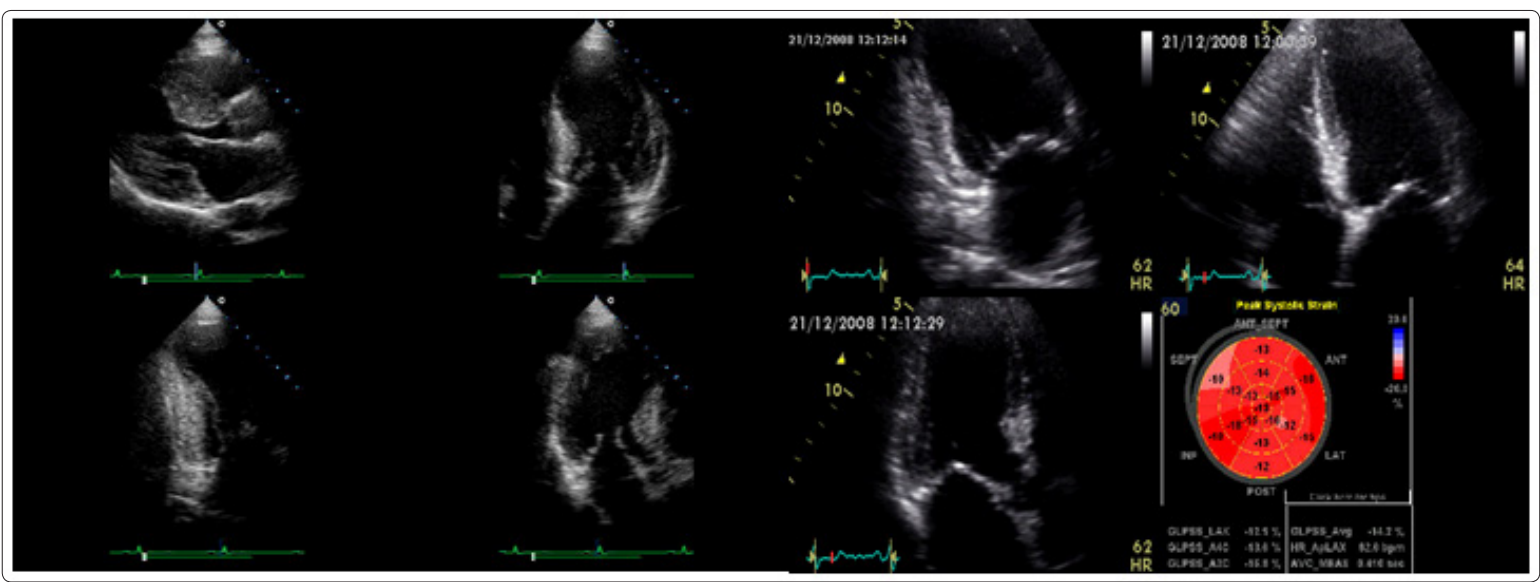

Figure 1: (A) Dobutamine Stress Echocardiography (DSE) : The role of DSE in the diagnosis of Low Flow Aortic Stenosis. The images in figure 1 (A) Show different acquisitions during DSE (Parasternal LAX, Apical 4 chamber view, Apical 2 chamber and 3 chambers views).(B) 2D Strain, GLS generated from apical 3 projection, which was taken at peak stress during of DSE. Protocol for uses a low-dose protocol, starting at $5 \mathrm{lg} / \mathrm{kg} / \mathrm{min}$ with an incremental increase every 3 min to a maximum dose of 10-20 ug/ $/ \mathrm{kg} / \mathrm{min}$. Once positive result is obtained (more than $20 \%$ increase in stroke volume (SV)), or an increase in AS jet velocity $\geq 4.0 \mathrm{~m} / \mathrm{s}$ and a mean gradient $(\geq 30-40 \mathrm{~mm} \mathrm{Hg})$, then the infusion should be stopped provided that valve area does not exceed $\left(1.0 \mathrm{~cm}^{2}\right)$ at any flow rate. It is very important to be very cautious and terminate immediately the dobutamine infusion when significant tachycardia happened (rise of heart rate more than 20 beat per minute over baseline), or when hypotension, or significant ventricular or supraventricular arrhythmias and symptoms occur. Doppler data are recorded at each stage and the report of the DSE study should include LVOT diameter, AS velocity, mean gradient, SV and valve area at each stage. LVEF should be measured at base line-resting state and peak effect. (Taken from reference (26 and 39)).

\section{Low Flow, Low Gradient As With Preserved Ejection Fraction (Paradoxical Low Flow As)}

The echocardiographic assessment of the severity of aortic valve stenosis (AS) usually relies on three main parameters including peak velocity, mean pressure gradient (MPG) and AVA, which should be concordant in the majority of aortic valve disease cases. In up to $20-30 \%$ of patients, these parameters are discordant (AVA $<1 \mathrm{~cm}^{2}$, AV velocity $<4.0 \mathrm{~m} / \mathrm{s}$ and $\mathrm{MPG}<40 \mathrm{mmHg}$ ). In most cases, these patients present with a stroke volume index $\geq 35 / \mathrm{ml} /$ $\mathrm{m}^{2}$ (average normal flow). For better assessment of these patients, direct visualization of AVA by trans esophageal echocardiography (TEE) and calcium score should be measured using computed tomography (CT) which could be of great help in these category of patients. Intervention is recommended in symptomatic patients with proven severe AS and low gradient, as for patients with classic severe AS [29, 30]. The sub-group of 'paradoxical' low flow, low gradient AS with preserved EF refers to patients with left ventricular hypertrophy $(\mathrm{LVH})$ resulting in reduced trans valvular flow ( $\mathrm{SV}$ index $<35 \mathrm{~mL} / \mathrm{m}^{2}$ ) despite normal LVEF [30].

The most demanding area in clinical setting is AVA $<1 \mathrm{~cm} 2$ with a lower peak velocity of $<4 \mathrm{~m} / \mathrm{s}$ and a MPG $<40 \mathrm{mmHg}$ despite normal LVEF. However, technical errors in AVA calculation (as LVOT diameter measurements) have to be carefully excluded, before the definite diagnosis of paradoxical low flow severe AS with preserved LVEF. Severe low flow low gradient AS with preserved LVEF has been described typically in elderly patients with LVH (small volume) and associated with Doppler and tissue Doppler evidence of LV diastolic dysfunction. In addition, reduced LV global longitudinal systolic strain (GLS) and fibrosis have been found in many cases. However, the majority of these patients had a history of long standing arterial hypertension that may also have caused the LV remodeling (hypertrophy and fibrosis) $[29,30]$. Importantly, diastolic function indices should be initially assessed non-invasively by conventional mitral Doppler flow, tissue Doppler imaging (TDI), filling pressure left atrial pressure $(\mathrm{E} / \mathrm{Ea})$ and pulmonary venous flow pattern.

Currently, it remains difficult in distinguishing between pseudo severe and true severe AS in clinical settings. The degree of valve calcification assessed by non-contrast $\mathrm{CT}$ may in this situation be an important hint to identify true severe AS [31]. However, only a high calcium score can ascertain severe AS while a low score makes severe AS highly unlikely. Moreover, careful follow up and reevaluation of symptomatic patients are recommended. In severe paradoxical low flow AS with preserved LVEF, patients are usually characterized by extensive LV concentric remodeling leading to a small LV cavity, restrictive LV filling pattern, and reduced systolic longitudinal myocardial shortening. 2DE by using conventional transthoracic approach (TTE) can be able to identify clearly the LV remodeling and different forms of hypertrophic cardiomyopathies in association with low-flow AS [32]. Similarly to the previous studies, the investigators found that echocardiographic calculation of AVA is highly dependent on accurate measurement of the diameter of the LVOT. To confirm the severity of AS, TEE should be used to measure the MPG, peak velocity and facilitate direct AVA planimetery area measurement in patients with poor TTE images, [33].

In patients with a valve area $<1.0 \mathrm{~cm}^{2}$ with low MPG $(<40$ $\mathrm{mmHG}$ ) and a velocity $<4 \mathrm{~m} / \mathrm{s}$ further classification based on the combination of velocity (gradient), trans valvular SV, and LVEF.

2DE and 3DE can also underestimate SV measurements which depend on LV end-diastolic, end-systolic volumes and LVOT geometry. Accordingly, multiple alternative approaches are recommended for calculation of SV index in clinical practice. Furthermore, there is an explanation for the low flow state which is most often due to a small ventricles with LVH and small LV volumes or LV systolic dysfunction with a reduced LVEF. In 
clinical decision-making, the diagnosis of severe AS requires a number of multidisciplinary approaches. These include (a) Clinical judgment such as the symptoms of the patients, (b) Imaging of valve anatomy and morphology with proper LVOT measurement using 2DE, 3DE, TEE and CT, (c) Illustrative evaluation of haemodynamic parameters employing Doppler results such as MPG, SV and velocities, (d) LV morphology assessment (remodelling) and proper evaluation of LV systolic function including LVEF, GLS, MPI and LV dP/dtmax ) and (e) diastolic function indices such as conventional Doppler velocities, TDI and pulmonary venous flow pattern [32, 34].

Although, the AS velocity and mean gradient may be lower than expected for a given valve area (low flow AS) in the presence of a small hypertrophied LV (which ejects a small SV), even when severe AS is present. Continuity-equation valve area is accurate and indexed AVA is of great help in this situation (indexing valve area to body size).

\section{Assessment of the Left Ventricular (LV) Morphology and Function in AS}

LVH and changes in LV function in response to AS are important for AS grading and the definition of the pathophysiological stage of disease. Similarly, LV remodeling and myocardial dysfunction caused by concomitant disease such as arterial hypertension (HTN), coronary artery disease (CAD) and cardiomyopathy may significantly affect AS assessment. These associated diseases can affect both LV systolic and diastolic functions which could reduce the SV / SV index and pressure-gradient in AS. It is important to emphasize here, that GLS by STE can differentiate between normal, ischemic-although viable (myocardial hibernation) and scarred or infarct myocardium in a quantitative manner. A MultiCentre trial is a pre-request before clinical application can be considered for scar-infarct. This can only be done once GLS shows systolic lengthening or zero systolic strain as well as an ischemic-viable myocardium which displays only a depressed systolic strain value. One important conclusion that emerges from the previous studies reviewed in this paper is that quantitative assessment of LV myocardium remodeling is an essential tool for integrating the wealth of information required to understand this complex system. The information can also help in predicting the impact of novel diagnostic modalities and proper therapies on a dysfunctional myocardium which undergoes remodeling following either myocardial infarction or left ventricular hypertrophy in AS hypertensive patients.

\section{Conventional Parameters of LV Function Assessment}

Conventional parameters of LV function include the measurement of LV dimensions at end-diastole and end-systole providing fractional shortening (FS) and end-diastolic and end-systolic volumes providing LVEF and SV. Because LVEF is load dependent, the increase in LV afterload in the presence of severe AS may result in apparent depression of LV systolic function indices which is manifested by low LVEF (measured by conventional 2DE) despite preserved LV myocardial function . So, correction of this afterload mismatch by AV replacement could improve LVEF and FS after relief of obstruction.

New Insight in the Assessment of LV Function In Paradoxical Low Flow As With Normal Ef Associated With Novel Parameters of LV Function Assessment

Alternative to LVEF, the following techniques are able to assess LV contractile function accurately in aortic and mitral valve diseases in patients with normal LVEF:-

i. Mitral annular displacement by tissue Doppler Imaging (TDI) ii. Global Longitudinal Systolic Strain (GLS)

iii. Myocardial Performance index (MPI) or (Tei Index) .

iv. BNP levels

v. Doppler-derived rates of left ventricular pressure rise and fall $(\mathrm{dP} / \mathrm{dt}$ and $-\mathrm{dP} / \mathrm{dt})$

In this State-Of-The-Art Review, It is Relevant to Focus on the Update in GLS, MPI and dP/dt.

Global Longitudinal Strain (GLS)

In severe AS, GLS measurements are able to detect sub clinical impairment of LV myocardial systolic function when EF is still normal and may predict prognosis in many studies [3,8,35-37]. In patients with aortic stenosis, GLS progressively worsens with increasing disease severity (from $-18.2 \pm 2.1 \%$ in mild to $-13.3 \pm 3.7 \%$ in severe aortic stenosis) [3]. Longitudinal strain has been shown to be a strong predictor of all-cause mortality independent of stenosis severity and LVEF or other known major risk factors of CAD and predictors of HF [37,38]. The observed changes in the shape and size of cardiac myocytes are considered cardiac remodeling, which are known to explain cardiac dysfunction in heart failure but may be physiological. These pathophysiological markers of pre heart failure (PHF) could be examined via GLS and other myocardial strains [32,37]. In clinical practice, GLS and area strain have been shown to be more reproducible and more useful compared to radial and circumferential strains. They have been demonstrated to be as accurate as son micrometry and magnetic resonance imaging (MRI) in several conditions related to cardiovascular diseases. 2D and 3D speckle tracking echocardiography have been used to quantify regional alterations of longitudinal strain and area strain through their polar projection, which allows a further evaluation of the exact site and extent of myocardial damage that may be important in clinical decision-making [37,38]. Furthermore, $2 \mathrm{D}$ and $3 \mathrm{D}$ speckle tracking echocardiography can overcome certain limitations in conventional 2DE in the assessment of cardiac function in AV disease [36,39]. Importantly, there is an evidence of negative Impact of myocardial fibrosis in patients with symptomatic severe AS which is associated with un favorable outcomes and may be reflected in reduced GLS [32,4043]. However, certain limitations to the clinical utility of GLS include some degree of afterload dependence and inter-vendor differences in strain measurements [4,44]. Although, the role of GLS in a symptomatic AS for the detection of preclinical stage of myocardial dysfunction is well defined, its clinical application as a parameter that may decide timing of intervention remains insufficiently defined. Nevertheless, GLS can be used as an additional imaging technique within an integrated approach to assess AS severity and its impact on LV myocardial function, in many occasions in selected patients as in (a) Paradoxical low flow severe AS with preserved LVEF , (b) Severe mitral and aortic valve incompetence lesions and in (c) Arterial hypertension in association with LVH . Interestingly, an excessive LVH as assessed by 2DE has been shown to be a predictor of outcome [45]. Although, accurate assessment of LV myocardial fibrosis is definitely requires cardiac MRI with late Gadolinium. A recent meta-analysis study [46] demonstrated that in a symptomatic patients with 2DE evidence of severe valvular AS and normal LVEF, impaired left ventricular GLS is associated with high mortality. These data emphasize the potential usefulness of LV GLS for risk stratification and early management of these patients. This meta- analysis included 1,067 a symptomatic patients with significant AS and LVEF $>50 \%$ were analyzed. The median of LV GLS was $-16.2 \%$. There were 91 deaths reported during followup with median of 20 Months, resulting in a pooled mortality rate of $8.5 \%$. The LV GLS performed well in the prediction of 
death . The best cut off value identified in this study was LV GLS of $-14.7 \%$. Random effective models have been employed in this meta-analysis and they demonstrated that the risk of death for AS patients is significant at LV GLS $<14.7 \%$; $p<0.0001$ ). Interestingly, the relationship between LV GLS and mortality remained significant in patients with normal LVEF $(\geq 60 \%), p=$ 0.001. Similarly, Lancellotti et al [41] examined a cohort of 163 patients with at least moderate to severe, a symptomatic AS. They demonstrated that impaired GLS was an independent predictor of survival. Those patients with GLS $>15.9 \%$ had significantly better outcome than patients with the strain less than $15.9 \%$ (4year survival of $63 \%$ vs. $22 \%, \mathrm{P}<0.001$ ). Interestingly, a recent publication in 2020 conducted by Elkilany et al [47], illustrated a concomitant dilated cardiomyopathy which was observed in $1.8 \%$ of subjects with bicuspid aortic valve (BAV). A few different clinical and echocardiographic characteristics were found. They concluded that, the presence of cardiomyopathy was independently associated with heart failure either clinically or at the sub clinical stage, which can be identified by global systolic strain at the preclinical stage of the disease. Furthermore, there are increasing role of GLS in the diagnosis of subclinical systolic dysfunctionand normal EF in arterial hypertension, $\mathrm{CAD}, \mathrm{HCM}$ and valvular heart diseases. Different studies have described the crucial role of three dimensional strain and 2D strain for detection of subclinical LV dysfunction and normal EF in hypertensive patients with an associated AS and paradoxical low flow-low gradient and severe AS. In addition, the presence of diastolic dysfunction (grade II-IV) and high left atrial pressure by conventional Doppler and TDI (Ea $<8 \mathrm{~cm} / \mathrm{s}, \mathrm{E} / \mathrm{Ea}>14)$, can clearly point to the mechanism(s) of low flow and low gradient AS with preserved EF [45-46].

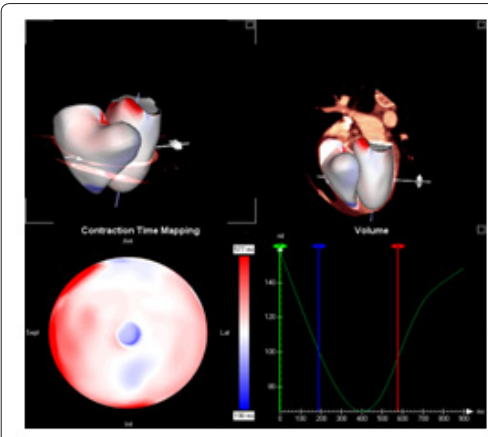

(A)

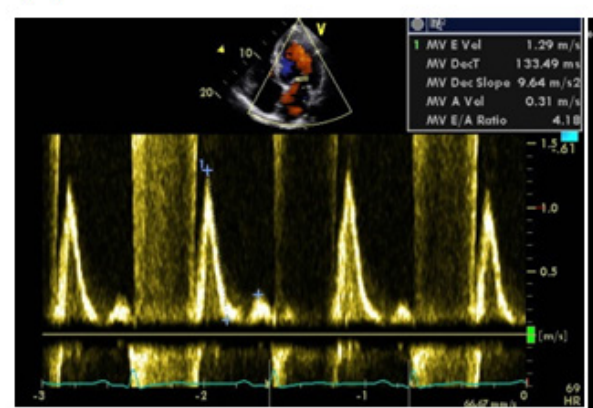

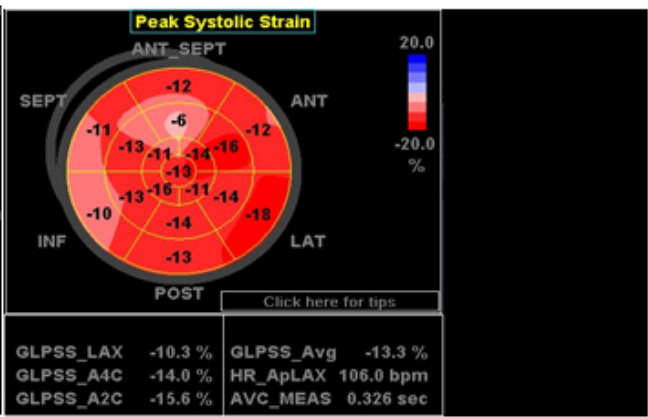

(B)

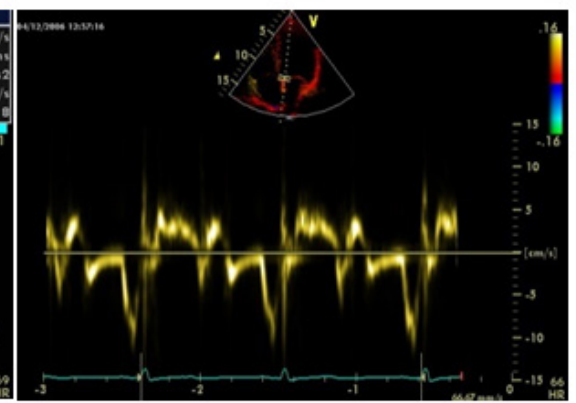

(D)

Figure 2: This figure illustrates the role global longitudinal systolic strain (GLS) in the diagnosis of subclinical systolic dysfunctionand normal EF in arterial hypertension. (A) shows 3D Strain and (B) shows 2D Strain for detection of subclinical LV dysfunction and normal EF (GLPSS-13\%), in hypertensive patient with low GLS in AS patient with paradoxical low flow- low gradient severe AS. The image in (C) shows grade III/IV diastolic dysfunction by conventional Doppler flow and the image in (D) shows evidence of diastolic dysfunction by tissue Doppler imaging $(\mathrm{Ea}<5 \mathrm{~cm} / \mathrm{s})$. Permission for the images was given by the corresponding author Dr. GEN Elkilany and from Dr. Bernhard Mumm, CEO Tom Tech Company, Germany.

In summary, the diagnosis of severe AS in clinical practice must be based on multidisciplinary and an integrated approach including : (i) Doppler assessment of trans-valvular velocity/ gradient,(ii) AVA (iii) Imaging of valve morphology,(iv) Proper assessment of flow rate and stroke volume (SV) (v) Evaluation of LV morphology (remodeling and LVH), (vi) Measurements of LV systolic and diastolic function indices by 2DE, TDI and GLS, (vii) Accurate measure of blood pressure level and finally (viii) Clinical assessment of the symptomatic status . Accordingly, it is essential in clinical settings to use an integrative approach when grading the severity of AS, combining all Doppler and 2DE data as well as clinical presentation, and not relying on one specific parameter. Optimization of loading conditions (arterial hypertension and high cardiac output states) which influence velocity and pressure gradients are crucial and therefore, these parameters which vary depending on illness of patients with low versus high cardiac output, should be considered in evaluation of valve disease severity. In addition, cardiac arrhythmias or tachycardia can interfere with proper Doppler assessment of AS severity. Consequently, hemodynamic assessment should be performed at an average blood pressures and heart rate levels within the normal range.

In conclusion, the implication of cardiac ultrasound with routine use of GLS is a must for early detection and proper management of cardiac dysfunction in a symptomatic AV disease patients and this is reasonably recommended in BAV and AS with concomitant AR [47]. 


\section{Myocardial Performance Index (MPI)}

Doppler-derived myocardial performance index (MPI) has been considered as a diagnostic and prognostic Doppler marker for many different clinical conditions. Tissue Doppler imaging MPI and Pulsed wave Doppler MPI were significantly higher in patients with grade I diastolic dysfunction than in control subjects: $0.49 \pm 0.14 \mathrm{vs}$. $0.40 \pm 0.09(P<0.001)$ and $0.45 \pm 0.11$ vs. $0.37 \pm 0.08(P<0.001)$, respectively. Cutoff values of TDI-MPI $>0.42$ and pulsed wave Doppler (PWD)-MPI $>0.40$ identified grade I diastolic dysfunction subjects, with good sensitivities and specificities in a recent study conducted by Jose M. et al [48]. Recently, Redfield et al studied the burden of systolic and diastolic ventricular dysfunction and demonstrated that, an initial diastolic dysfunction detected by Doppler echocardiography was an independent risk factor for the development of heart failure (HF) and all-cause mortality, even in a symptomatic patients [49]. The myocardial performance index (MPI) or Tei-Index, described more than a decade ago, has been well documented in the literature as a prognostic marker and can predict the progression of various cardiovascular diseases [20,50]. However, in the majority of these studies, MPI was used in patients with combined systolic and diastolic dysfunctions. Furthermore, Liu D et al demonstrated recently, the great value of tissue Dopplerderived Tei index and two-dimensional speckle tracking imaging derived longitudinal strain on predicting outcome of patients with light-chain cardiac amyloidosis [51].

An alternative for MPI calculation is the use of the pulsed-wave TDI-derived MPI, which allows simultaneous measurement of both the diastolic and systolic intervals in the same cardiac cycle, with high diagnostic accuracy in subjects with heart failure and LV myocardial dysfunction $[52,53]$. This new technique, can overcome the limitation of the conventional Doppler-derived MPI method which are based on flow-velocity curves and are performed in different cardiac cycles. An important study conducted by Johan and his colleagues [54] compared eight different echocardiographic and Doppler indices of cardiac function as predictors of congestive heart failure (CHF) using a population-based cohort of more than five hundred elderly a symptomatic men (without overt HF) and significant valve disease at baseline (median follow-up time 8.2 years). In a multivariable Cox proportional-hazard analysis including the different indices of cardiac function, high MPI, abnormal LV wall motion score index and diastolic dysfunction (a pseudo-normalized/restrictive- E/Aratio pattern) independently predicted future CHF morbidity. After adding traditional $\mathrm{CHF}$ risk factors (age, previous $\mathrm{CAD}$, diabetes mellitus, arterial hypertension, hyperlipidemia, smoking, LVH and body mass index) to the above model, only a high MPI remained a significant predictor $(\mathrm{p}=0.002)$. The investigators concluded that MPI can provide an important prognostic information for the risk of future congestive heart failure, beyond other measurements of diastolic and systolic cardiac function indices and traditional HF risk factors in 70 years elderly men [52-54].

\section{Aortic regurgitation (AR)}

Up to $80 \%$ of adults with AS also have an associated mild or moderate AR. Although, measures of AS severity are not significantly affected in mild to moderate AR lesions. However, in severe AR and because of the increased trans aortic valve volume flow rate, maximum velocity and MPG will be higher than expected for a given valve area. In this situation, reporting accurate quantitative data for the severity of both stenosis and regurgitation is helpful for clinical decision-making $[55,56]$. Furthermore, velocity and mean gradient still remain the major predictors of outcome in combined moderate AS and moderate AR [55]. Aortic regurgitation patients with symptomatic moderate-tosevere and severe aortic regurgitation have more impaired GLS than those without symptoms $(-14.9 \pm 3.0 \%$ vs. $-16.8 \pm 2.5 \%)$ respectively [57]. In asymptomatic patients, the risk of death continuously increases as GLS worsens [57]. In the same study, GLS significantly improved the reclassification of mortality risk and was independently associated with the need for aortic valve surgery in asymptomatic patients with moderately severe or severe aortic regurgitation and preserved LVEF $[57,58]$. In patients undergoing aortic valve surgery, preoperative GLS worse than $-19 \%$ was associated with reduced long-term survival after surgical procedure [59].

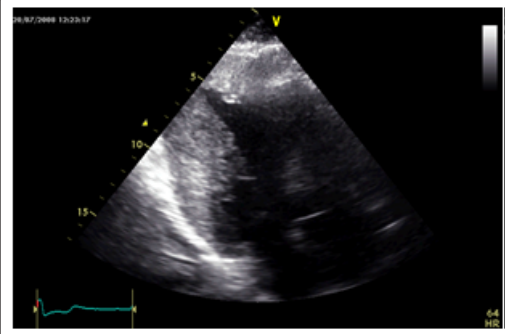

(A) Apical Hypertrophic cardiomyopathy

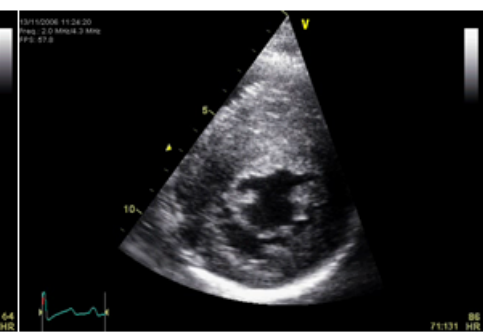

(B) HOCM

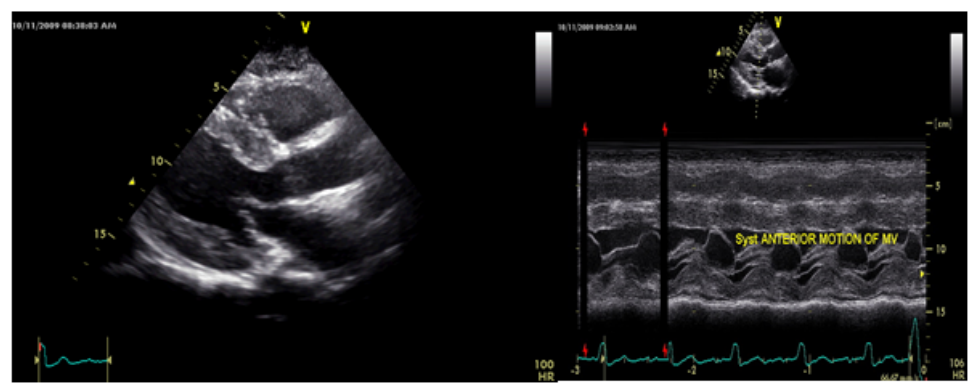

(C) Hypertensive HD with LVH

(D) Sub-aortic obstruction in HHD

Figure 3: Images A-D show the different forms of Hypertrophic Cardiomyopathies that can be responsible of paradoxical low flow aortic stenosis with normal EF. Abbreviation, HOCM, hypertrophic obstructive cardiomyopathy , HD , heart disease , HHD, hypertensive heart disease. 


\section{Mitral stenosis and right-sided valve diseases}

Recent studies suggested that strain echocardiography might be useful to improve the assessment of myocardial function in valve diseases, particularly in detecting subclinical dysfunction and defining the timing of interventions. However, a majority of studies focused on aortic and mitral valve disease, while data on right-sided valve diseases are limited. Patients with severe mitral stenosis have reduced LV deformation which is related to the severity of mitral stenosis [60]. Improvement in strain values was detected within 72 hours after balloon mitral valvuloplasty of severe mitral valve stenosis. The potential prognostic value of right ventricular (RV) longitudinal strain was found in patients with significant tricuspid regurgitation [61]. Impaired RV free wall longitudinal strain (worse than $-23 \%$ ) was associated with worse outcome with a prognostic value beyond conventional echocardiographic parameters of RV systolic function. In patients with pulmonary valve disease undergoing percutaneous pulmonary valve implantation, pre interventional RV longitudinal strain can predict improvement of exercise function after intervention [62].

\section{Mitral Regurgitation}

In patients with severe primary mitral regurgitation undergoing interventions, preoperative GLS is an independent predictor of cardiovascular events and death and appears to have incremental predictive value over conventional clinical and echocardiographic risk factors [25,63-66]. One of the largest studies revealed that mitral valve surgery in patients with a symptomatic severe mitral regurgitation was beneficial with respect to outcome (all-cause mortality), particularly in those with GLS worse than -21\% [64]. In addition, preoperative GLS worse than $-18.1 \%$ predicted post procedural LV dysfunction in patients undergoing mitral valve surgery [63]. Interestingly, the GLS reported in these studies was higher than what is considered the lower limit of normal, which indicates that strain values that are otherwise considered normal are already associated with impaired outcome in patients with primary mitral regurgitation. Large studies evaluating the clinical utility of strain in secondary mitral regurgitation are limited. Recently, a retrospective analysis of 650 patients with moderate and severe secondary mitral regurgitation demonstrated that GLS worse than $-7.0 \%$ was associated with increased risk for all-cause mortality, whereas LVEF was not [67]. The lower GLS found in this study compared to those with primary regurgitation may be explained with specific high-risk patient population that was investigated. The study population mostly comprised of patients with advanced heart failure with a mean LVEF of $29 \pm 10 \%$, half of which had an ischemic cardiomyopathy.

\section{Concomitant Mitral Regurgitation (MR) and Aortic Valve Disease}

MR is common in elderly adults with fibro calcific AS either as a consequence of increased LV pressure overload, or in association with concomitant mitral valve disease. These include in the following conditions : (i) Rheumatic heart disease, (ii) Degenerative etiology due to mitral valve calcification or extension of calcification from calcific aortic valve to mitral valve apparatus , (iii) Mitral valve prolapse, and (iv) Secondary MR etiologies (Ischemic MR and in dilated cardiomyopathy).

It is crucial to use standard approaches and utilization of different echocardiographic projections to distinguish MR owing to a primary abnormality of the mitral valve from secondary MR related to increased pressure overload (secondary to AS).

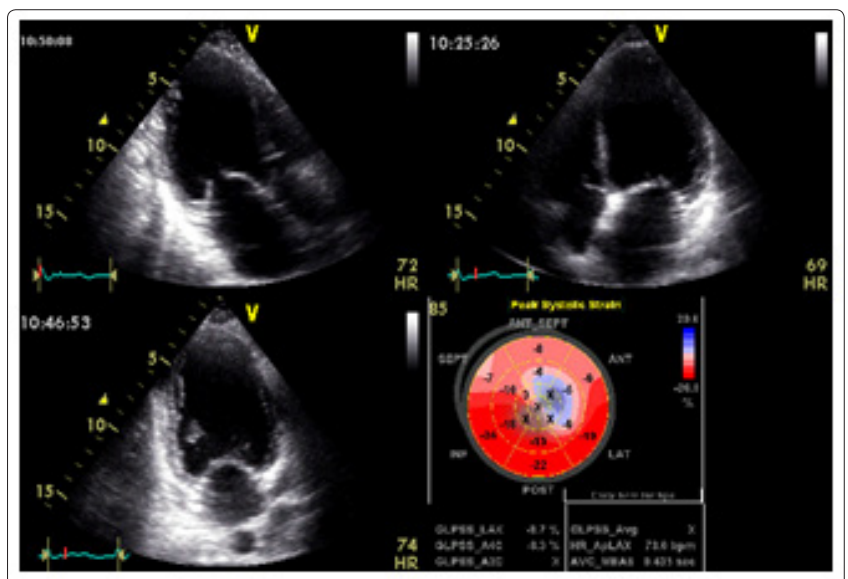

Figure 4: This figure illustrates the process of ischemic etiology of pseudo severe AS which is associated with other causes of LV dysfunction such as myocardial infarction. Note that depressed strain values appear at the basal septal and basal anterior wall segments as represented in the ischemic -viable myocardium (pink in color-light red). Similarly, systolic lengthening and 0 strain (blue in color) represent the infarct-scar at mid-antero-septal segments and apex proper of the left ventricle by GLS. These values are correlated to the regional wall motion abnormalities from hypokinetic to a kinetic myocardium respectively, at left anterior descending coronary artery (LAD). The territories are presented in 3 apical projections by $2 \mathrm{DE}$. All rights reserved to the corresponding author, GEN Elkilany.

Critical Points in Assessment of the Severity of MR and AS in the Presence of Concomitant AS with MR (Clinical Perspectives):

a) Timing of the signal and configuration of the slope velocity are the most reliable way to distinguish the CW Doppler velocity curve of MR from AS. Moreover, MR is longer in duration, starting with mitral valve closure and continuing until mitral valve opening. AS jet is of crescendo decrescendo contour, started just after MV closure and terminates before the second heart sound in the majority of cases.

b) In severe MR, trans-aortic flow rate may be low resulting in a low gradient even when severe AS is present with valve area calculations remain accurate in this setting as long as flow is calculated in the LVOT.

c) High LV pressure due to severe AS, may lead to MR severity overestimation .The high driving pressure will also disproportionately increase the MR volume for a given regurgitated orifice area.

d) Furthermore, it is essential for the clinical decision making to decide whether the mitral valve requires intervention in the setting of an associated aortic valve disease (AS) by careful evaluation of the mechanism (s) of MR and its severity. Evaluation of the grades of MR severity should include : (a) Effective regurgitant orifice area (EROA), (b) Regurgitant volume, (c) Regurgitant fraction (RF) and (d) Proximal isovelocity surface area (PISA) method [55,56, 68].

\section{Arterial Hypertension (HTN)}

Earlier studies described HTN which was accompanied AS in $35-45 \%$ of patients. However, in elderly patients with low flow, low gradient AS, it was present in the majority of these patients $[29,30]$. The presence of HTN may primarily affect flow and gradients but far less AVA measurements. Impaired GLS in arterial hypertensive patients was detected in many studies with or without a concomitant MR or aortic valve diseases, although 
the LVEF was still remaining at the normal range (> 50\%) $[32,64-$ 67]. Nevertheless, in uncontrolled severe arterial hypertension, the measure of grade of aortic valve stenosis severity may not be accurate (can make MPG and velocity changes) and will not reflect the exact AS severity [69-70]. Thus, control of blood pressure is a must before echocardiographic evaluation.

\section{Echocardiographic Predictors of Symptom Development and Adverse Outcomes in AS [3,7,71,72]}

The followings are the criteria that increase the likelihood of severe AS in patients with AVA $<1.0 \mathrm{~cm} 2$ and mean gradient less than expected $(<40 \mathrm{~mm} \mathrm{Hg})$ in the presence of preserved EF:-

1. Clinical criteria:- Physical examination consistent with severe aortic valve disease, typical triad symptoms of (chest pain, syncope and dyspnea) without other explanation.

2. Qualitative imaging data:-The presence of otherwise unexplained LVH, reduced GLS without other explanation, an increased echo reflection (echo-dense aortic valve) due to extensive calcification, limited or no cusp motion and a decrease in the maximal aortic cusp separation in $2 \mathrm{DE}$ and M-Mode.

3. Quantitative multimodality imaging techniques (2DE, CW and PW Doppler, CT, cardiac MRI): Mean gradient 30-40 $\mathrm{mm} \mathrm{Hg}, \mathrm{AVA}<0.9 \mathrm{~cm} 2$ and low flow (SV index $<35 \mathrm{~mL}$ / $\mathrm{m} 2)$ :- Low flow low gradient AS should be confirmed by other techniques than standard Doppler technique (LVOT measurement by 3D TEE or calcium score by CT, cardiac MRI or/and invasive data) .

A recent study revealed that a symptomatic patients with severe aortic stenosis undergoing treadmill stress echocardiography, lower LV GLS were associated with higher longer term mortality and offer incremental prognostic value [73]. Similarly, in symptomatic severe AS, either high-gradient or low-flow, low-gradient, preoperative GLS was associated with long-term postoperative cardiac mortality and morbidity in all therapeutic approaches in either surgical or trans catheter aortic valve replacement $[74,75]$ . In a symptomatic severe AS and normal EF, a GLS worse than $-18.2 \%$ has been shown to be associated with disease progression, evidenced by symptoms onset and need for valve intervention [76]. In a recent meta-analysis including 1067 patients, a GLS worse than $-14.7 \%$ predicted occurrence of death with $60 \%$ sensitivity and $70 \%$ specificity and was associated with a $>2.5$ fold increased risk of death [77]. Interestingly, the Heart Valve Clinic International Database (HAVEC) group recommended the incorporation of GLS into therapeutic decision-making [78]. Thus, patients with a symptomatic severe aortic stenosis with GLS worse than $-16.0 \%$ and other high-risk factors (high-calcium score on cardiac computer tomography and myocardial fibrosis detected by magnetic resonance) may be considered for intervention. A similar algorithm was recently proposed by Dahl et al. [79].

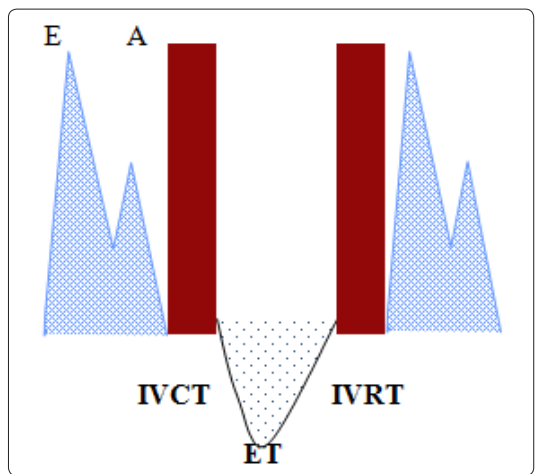

Figure 5: A Schematic diagram showing how to measure index of myocardial performance (MPI) : isovolumetric relaxation time(IVRT), isovolumetric contraction time (IVCT), LV ejection time (ET), MPI= IVRT+IVCT/LVET.E, points to early mitral Doppler diastolic velocity; A represent the pre systolic-late mitral Doppler diastolic velocity filling pattern.

\section{Maximum rate of $L V$ Pressure Development ( $L V \mathrm{dP} / \mathrm{dT})$ : The Role in a Preclinical Detection of LV Myocardial Dysfunction in VHD}

The Doppler-derived rates of LV pressure rise $(\mathrm{dP} / \mathrm{dt})$ has important clinical implication.

Interestingly, a consensus published by Cecconi $\mathrm{M}$. and his colleagues revealed that peak velocity of the early diastolic wave and rate of $\mathrm{LV}$ pressure drop $(-\mathrm{dP} / \mathrm{dt})$ were identified as independent predictors of CHF. The findings suggested that Doppler-derived $\mathrm{dP} / \mathrm{dt}$ and $-\mathrm{dP} / \mathrm{dt}$, used in combination with conventional echocardiographic variables, could allow a better understanding of myocardial dysfunction and a possibility for prediction of the risk of heart failure in dogs with chronic mitral valve disease [80].

Furthermore, the investigators demonstrated that LV contractility (inotropic state of the left ventricle) is one of the main determinants of cardiac function and is considered as an important element of the hemodynamic evaluation of the critically ill patients [80]. LVEF was estimated by 2DE as the fractional area of contraction. However, LVEF has known limitations as an index of cardiac systolic (inotropic) function, such as the high dependency on the cardiac loading conditions [81]. Although new echocardiographic indexes, such as speckle-tracking-derived LV global longitudinal strain or strain rate, have been recently introduced, their need of sophisticated software, standardization of methodology and well trained operators preclude their use for continuous hemodynamic monitoring of the LV systolic function in critically ill patients. Alternatively, the maximum rate of LV pressure during isovolumetric contraction $\left(\mathrm{LV} \mathrm{dP} / \mathrm{dt}_{\max }\right)$ has been classically considered as a marker of LV inotropic state $[82,83]$. Left ventricular $\mathrm{dP} / \mathrm{dt}_{\max }$ has been traditionally used as a reliable marker of myocardial performance $[83,84]$. The maximal rate of $\mathrm{LV}$ pressure rise $\left(+\mathrm{dP} / \mathrm{dt}_{\text {max }}\right)$ is usually developed during isovolumetric contraction under normal circumstances [84], so theoretically $\mathrm{LV} \mathrm{dP/dt}{ }_{\text {max }}$ should be relatively insensitive to afterload.

\section{Advantages of Systolic Index of Contractility (DP/DT)}

The maximum rate of rise of LV pressure during the isovolumic contraction phase of $\mathrm{LV}$ systole, $\mathrm{dP} / \mathrm{dt}\left({ }_{\max }\right)$ is considered a sensitive and good measure of LV contractility. This technique is not affected by afterload and very minimally influenced by preload [84]. The prerequisite for this measurement using 2D and Doppler echocardiography is that the associated mitral valve should have some degree of central mitral valve regurgitation (MR). In that case, the shape of the MR jet, which should be interrogated with a continuous wave Doppler (CWD) and the spectral display optimized, reflects the instantaneous pressure difference between the LV and LA during LV systole.

In the presence of global LV dysfunction, the LV pressure buildup will decrease and the LA pressure will increase which will decrease the rate of rise of MR jet velocity. This is the principle behind this method of assessing global LV function. [64,84]. In order to calculate $\mathrm{dP} / \mathrm{dt}\left({ }_{\max }\right)$, the time taken for the velocity to rise from $1 \mathrm{~m} / \mathrm{s}$ to $3 \mathrm{~m} / \mathrm{s}$ during measurement by CWD (of MR jet during isovolumetric contraction phase) using the modified Bernoulli equation $[64,84]$. However, there are some limitations in clinical 
application of $\mathrm{dP} / \mathrm{dt}\left({ }_{\max }\right)$ which include the followings:-

1. A good MR signal is mandatory which may not always be the case. Even eccentric jets which will not be optimally aligned to the CW Doppler are of no use.

2. A small error in the time interval will produce a large change in $\mathrm{dP} / \mathrm{dt}(\max )$ value because we are dividing a large number, i.e., 32,000 by the time interval.

3. This method is inappropriate in the presence of acute MR because of high left atrial pressures.

4. $\mathrm{dP} / \mathrm{dt}(\max )$ is not an entirely load-independent index of LV function.
Moreover, in the presence of LVH secondary to aortic valve disease, the speed of left-heart relaxation is impaired, but the peak rate of myocardial relaxation is unaltered. Abnormalities of LV systolic function have no direct bearing on relaxation, and the extent of LVH appears to be a determinant of left-heart relaxation. [85] However, for future studies, multimodality cardiac imaging techniques can be utilized for a proper assessment of cardiac function and patients at risk of heart failure at the preclinical stage of the disease $[37,64,86]$.

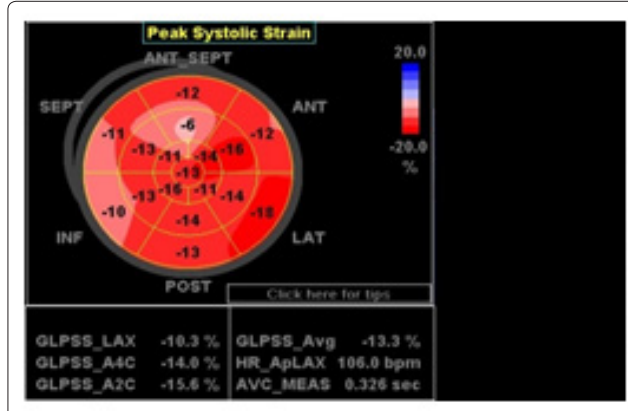

(A)

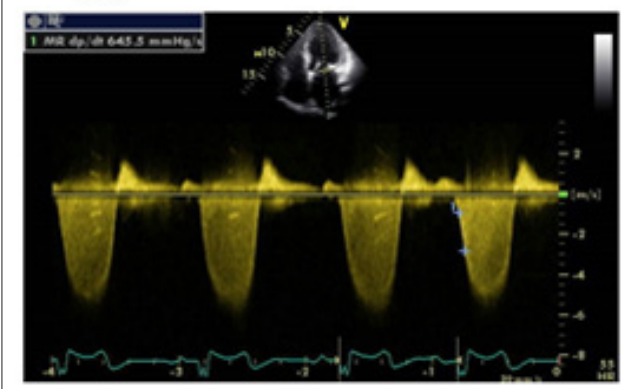

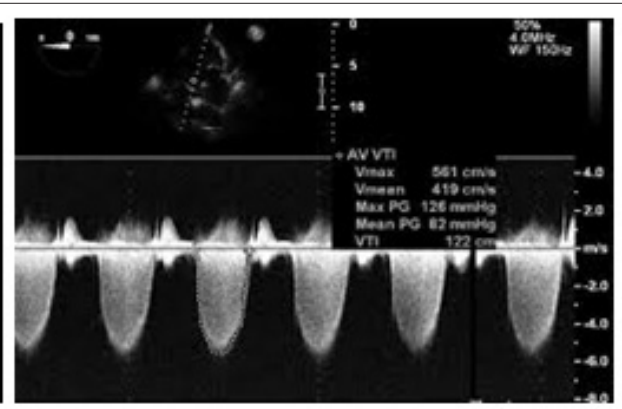

(B)

(C)

Figure 6: (A) Bulls eye technique (automated function imaging algorithm), GLS generated from apical 2,3 \& 4 chamber views illustrated the impaired global longitudinal peak systolic strain (GLS) of $-13 \%$ in hypertensive patient and significant mitral valve incompetence and AS, but normal ejection fraction ( $\mathrm{EF}=51 \%$ ), (B) Shows severe AS in correlation to GLS in (A). (C) shows that maximum rate of max. LV positive pressure development $(\mathrm{MR}+\mathrm{dp} / \mathrm{dt})$, which is depressed in the same patient $(645 \mathrm{~mm} \mathrm{Hg} / \mathrm{s})$. This figure also illustrates, how to measure $\mathrm{dp} / \mathrm{dt}$, at $1^{\text {st }}$ and $3^{\text {rd }} \mathrm{m} /$ seconds of MR CWD slope (Taken from reference [64]).

Table 1: Data showing $\mathrm{LV}$ function and the changes in $\mathrm{dP} /$ $\operatorname{dt}(\max )$ over time

\begin{tabular}{|l|l|}
\hline LV function & $\begin{array}{l}\mathbf{d P} / \mathbf{d t} \\
(\mathbf{m m H g} / \mathbf{s})\end{array}$ \\
\hline Normal & $>1200$ \\
\hline Borderline & $800-1200$ \\
\hline Reduced & $<800$, severely reduced $<500$ \\
\hline
\end{tabular}

\section{Conclusion}

Echocardiographic strain imaging allows for new insight into a complex cardiac mechanics and enables more precise evaluation of cardiac function. Hence, it has been shown to have clinical utility in a variety of VHD. In particular, global longitudinal strain has been shown to be more sensitive to detect systolic dysfunction than left ventricular ejection fraction. Subclinical myocardial dysfunction that is characterized by impaired LV GLS is often present in patients with a symptomatic severe VHD and in severe AS with preserved LVEF. Left ventricular GLS further deteriorates over time and impaired GLS at baseline is associated with an increased risk for progression to the symptomatic stage and the need for aortic valve intervention in severe AS and AR patients. Similarly, depressed LV GLS in a symptomatic severe MR could identify high risk patients which could benefit from early mitral valve repair or replacement. Strain echocardiography and MPI in VHDs have been demonstrated to be a useful complementary echocardiographic method that can identify patients at risk of developing either symptoms or poor survival and might assist in therapeutic decision-making. In addition, MPI seems to be a clinically relevant monitoring of cardiac function and may prove to be a valuable tool in assessing the risk of future congestive HF. In patients with valvular heart diseases GLS can provide both diagnostic and prognostic information in addition to standard echocardiographic and clinical parameters. However, a profound understanding of the complex interaction between loading conditions, chamber geometry, and contractility is necessary for the correct interpretation of myocardial deformation in order to draw appropriate conclusions in patients with valve disease.

Finally, the maximum rate of $\mathrm{LV}$ pressure rise ( $\left.\mathrm{LV} \mathrm{dP} / \mathrm{dt}_{\max }\right)$ has been traditionally used as a reliable marker of myocardial performance. Although a multicenter trials are required for a 
potential use and clinical application of $\mathrm{LV} \mathrm{dP/ \textrm {dt } _ { \operatorname { m a x } }}$, theoretically,

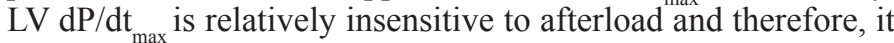
can be used as a sensitive method for myocardial contractility in patients with valvular heart disease . A prerequisite for this measurement using 2D and Doppler echocardiography is that the associated mitral valve should have some degree of central MR for proper assessment.

\section{Conflict of interest: None declared.}

\section{References}

1. Mor-Avi V, Lang RM, Badano LP, Belohlavek M, Nuno Miguel Cardim, et al. (2011) Current and evolving echocardiographic techniques for the quantitative evaluation of cardiac mechanics: ASE/ EAE consensus statement on methodology and indications endorsed by the Japanese Society of Echocardiography. Eur J Echocardiogr 12: 167205.

2. Voigt JU, Pedrizzetti G, Lysyansky P, Tom H Marwick, Helen Houle et al. (2015) Definitions for a common standard for 2D speckle tracking echocardiography: consensus document of the EACVI/ ASE/Industry Task Force to standardize deformation imaging. Eur Heart J Cardiovasc Imaging; 16: $1-11$.

3. Vollema EM, Sugimoto T, Shen M, Lionel Tastet, Arnold C. $\mathrm{T}$. Ng, et al. (2018) Association of Left Ventricular Global Longitudinal Strain With Asymptomatic Severe Aortic Stenosis: Natural Course and Prognostic Value. JAMA Cardiol 3: 839-847.

4. Ünlü S, Mirea O, Duchenne J, Efstathios D Pagourelias, Stéphanie Bézy, et al. (2018) Comparison of Feasibility, Accuracy, and Reproducibility of Layer-Specific Global Longitudinal Strain Measurements Among Five Different Vendors: A Report from the EACVI-ASE Strain Standardization Task Force. J Am Soc Echocardiogr 31: 374-380.

5. Dobrovie M, Bėzy S, Ünlü S, et al. (2019) How Does Regional Hypertrophy Affect Strain Measurements With Different Speckle-Tracking Methods? J Am Soc Echocardiogr; 32: 1444-1450.

6. Ünlü S, Duchenne J, Mirea O, Monica Dobrovie, Stėphanie Bèzy, et al. (2020) Impact of apical foreshortening on deformation measurements: a report from the EACVI-ASE Strain Standardization Task Force. Eur Hear J Cardiovasc Imaging 21: 337-343.

7. Baumgartner H, Falk V, Bax JJ, Michele De Bonis, Christian Hamm, et al. (2017) ESC Scientific Document Group. 2017 ESC/EACTS Guidelines for the management of valvular heart disease. Eur Heart J; 38: 2739-2791.

8. Ng ACT, Prihadi EA, Antoni ML, Matteo Bertini, See Hooi Ewe, et al. (2018) Left ventricular global longitudinal strain is predictive of all-cause mortality independent of aortic stenosis severity and ejection fraction. Eur Heart J Cardiovasc Imaging; 19: 859-867.

9. Marciniak A, Sutherland GR, Marciniak M, Piet Claus, Bart Bijnens, et al. (2009) Myocardial deformation abnormalities in patients with aortic regurgitation: a strain rate imaging study. Eur J Echocardiogr; 10: 112-119.

10. Marciniak A, Claus P, Sutherland GR, IvarSjaastad, Emil Knut Stenersen Espe (2020) Changes in systolic left ventricular function in iso- Cvijic and Voigt Strain in valvular disease; Anatol J Cardiol 23: 244- 252

11. Stokke TM, Hasselberg NE, Smedsrud MK, Sebastian I Sarvari, Kristina H Haugaa, et al. (2017) Geometry as a
Confounder When Assessing Ventricular Systolic Function: Comparison Between Ejection Fraction and Strain. J Am Coll Cardiol 70: 942-954.

12. Smedsrud MK, Pettersen E, Gjesdal O, Jan L Svennevig, Kai Andersen, et al. (2011) Detection of left ventricular dysfunction by global longitudinal systolic strain in patients with chronic aortic regurgitation. J Am Soc Echocardiogr; 24: $1253-1259$.

13. Dahl JS, Videbæk L, Poulsen MK, Torsten R. Rudbæk, Patricia A. Pellikka, et al. (2012) Global strain in severe aortic valve stenosis relation to clinical outcome after aortic valve replacement. Circ Cardiovasc Imaging 5: 613-20.

14. Alashi A, Mentias A, Abdallah A, Ke Feng, A. Marc Gillinov, et al. (2018) Incremental Prognostic Utility of Left Ventricular Global Longitudinal Strain in Asymptomatic Patients With Significant Chronic Aortic Regurgitation and Preserved Left Ventricular Ejection Fraction. JACC Cardiovasc Imaging 11: 673-682.

15. Kim HM, Cho GY, Hwang IC, Choi HM, Park JB, et al. (2018) Myocardial Strain in Prediction of Outcomes After Surgery for Severe Mitral Regurgitation. JACC Cardiovasc Imaging; 11(9): 1235-1244.

16. Vollema EM, Sugimoto T, Shen M, Tastet L, Ng AC, et al. (2018) Association of Left Ventricular Global Longitudinal Strain With Asymptomatic Severe Aortic Stenosis: Natural Course and Prognostic Value. JAMA Cardiol 3: 839-847.

17. Ewe SH, Haeck ML, Ng AC, Tomasz G Witkowski, Dominique Auger, et al. (2015) Detection of subtle left ventricular systolic dysfunction in patients with significant aortic regurgitation and preserved left ventricular ejection fraction: speckle tracking echocardiographic analysis. Eur Heart J Cardiovasc Imaging 16: 992-999.

18. Yasunobu H, Yukako H, Shoji K. (2019) A novel index equivalent to the myocardial performance index for right ventricular functional assessment in children and adolescent patients. SCIENTIFIC REPORTS , natureresearch; 9 (19975): $1-10$.

19. Redfield MM, Jacobsen SJ, Burnett JC Jr, Douglas W Mahoney, Kent R Bailey, et al. (2003) Burden of systolic and diastolic ventricular dysfunction in the community: appreciating the scope of the heart failure epidemic. JAMA 289: 194-202.

20. Niemann M, Breunig F, Beer M, Kai Hu, Dan Liu, et al. (2011) Tei index in fabry disease. J Am Soc Echocardiogr 24: 1026-32.

21. Liu D, Hu K, Herrmann S, Cikes M, Georg Ertl, et al. (2017) Value of tissue Doppler-derived Tei index and twodimensional speckle tracking imaging derived longitudinal strain on predicting outcome of patients with light-chain cardiac amyloidosis. Int J Cardiovasc Imaging 33: 837-845.

22. Meric M, Yesildag O, Yuksel S, et al. (2014)Tissue doppler myocardial performance index in patients with heart failure and its relationship with haemodynamic parameters. Int J Cardiovasc Imaging 30: 1057-1064.

23. Duzenli MA, Ozdemir K, Aygul N, Ahmet Soylu, Meryem Ulku Aygul, et al. (2009) Comparison of myocardial performance index obtained either by conventional echocardiography or tissue Doppler echocardiography in healthy subjects and patients with heart failure. Heart Vessel 24: 8-15.

24. Lancellotti P, Moonen M, Magne J, Kim O'Connor, Bernard Cosyns, et al. (2010) Prognostic effect of long-axis left ventricular dysfunction and B-type natriuretic peptide levels in asymptomatic aortic stenosis. Am J Cardiol; 105: 383-388. 25. Elkilany, GEN, De Groef M, Kabbash (2011) How to Identify 
Latent Systolic Dysfunction and Post-Operative Risk in Patients with Mitral Incompetence and Normal Ejection .World J Cardiovas. Surg.; 1(2) :11-17.

26. Nishimura RA, Grantham JA, Connolly HM, Hartzell V. Schaff, Stuart T. Higano, et al. (2002) Low-output, lowgradient aortic stenosis in patients with depressed left ventricular systolic function: the clinical utility of the dobutamine challenge in the catheterization laboratory. Circulation 106: 809-813.

27. Takeda S, Rimington H, Chambers J (1999) The relation between trans aortic pressure difference and flow during dobutamine stress echocardiography in patients with aortic stenosis. Heart 82: 11-14.

28. Monin JL, Que're' J-P, Monchi M, Hélène Petit, Serge Baleynaud, et al. (2003) Low-gradient aortic stenosis operative risk stratification and predictors for long-term outcome: a multicenter study using dobutamine stress hemodynamics. Circulation 108: 319-324.

29. Baumgartner H., Hung J., Edvardsen T, John B Chambers, Thor Edvardsen, et al. (2017) Recommendations on the Echocardiographic Assessment of Aortic Valve Stenosis: A Focused Update from the European Association of Cardiovascular Imaging and the American Society of Echocardiography. Eur Heart J Cardiovasc Imaging 18: 254-275.

30. Pibarot P, Dumesnil JG (2012) Low-flow, low-gradient aortic stenosis with normal and depressed left ventricular ejection fraction. J Am Coll Cardiol 60: 1845-1853.

31. Clavel M-A, Messika-Zeitoun D, Malouf J, Shivani R Aggarwal, Joseph Malouf, et al. (2013) The complex nature of discordant severe calcified aortic valve disease grading: new insights from combined Doppler echocardiographic and computed tomographic study. J Am Coll Cardiol 62: 2329-2338.

32. Elkilany, Galaleldin Nagib, Allah, Sherif Baath, Fedacko, et al. (2019) Hypertensive Cardiomyopathy: Diagnostic Approach and Clinical Differentiation from Hypertrophic Cardiomyopathy; J Cardiol \& Cardiovasc Ther 15: 1-8.

33. Chahal NS, Drakopoulou M, Gonzalez-Gonzalez AM, Ramasamy Manivarmane, Rajdeep Khattar, et al. (2015) Resting aortic valve area at normal trans aortic flow rate reflects true valve area in suspected low-gradient severe aortic stenosis. JACC Cardiovasc Imaging 8: 1133-1139.

34. Nishimura RA, Otto CM, Bonow RO, Blase A. Carabello, John P. Erwin, et al. (2014) AHA/ACC guideline for the management of patients with valvular heart disease: a report of the American College of Cardiology/American Heart Association Task Force on Practice Guidelines. Circulation 129 : 2440-2492.

35. Lancellotti P, Donal E, Magne J, Kim O’Connor, Marie L. Moonen, et al. (2010) Impact of global left ventricular afterload on left ventricular function in asymptomatic severe aortic stenosis: a two-dimensional speckle-tracking study. Eur J Echocardiogr 11: 537-543.

36. Kempny A, Diller GP, Kaleschke G, Stefan Orwat, Angela Funke, et al. (2013) Longitudinal left ventricular 2D strain is superior to ejection fraction in predicting myocardial recovery and symptomatic improvement after aortic valve implantation. Int J Cardiol 167: 2239-2243.

37. Ram B Singh, Fedacko Jan, Galal E Nagib Elkilany (2020) Guidelines on Pre-Heart Failure in the Light of 2D and 3D Speckle Tracking Echocardiography. A Scientific Statement of the International College of Cardiology. World Heart J. WHJ 12: 1-20.

38. Kusunose K, Goodman A, Parikh R, Tyler Barr, Shikhar
Agarwal, et al. (2014) Incremental prognostic value of left ventricular global longitudinal strain in patients with aortic stenosis and preserved ejection fraction. Circ Cardiovasc Imaging 7: 938-945.

39. Hatim Seoudy (2020) Limitations of echocardiography in the assessment of aortic valve disease.-Journal of Cardiology Practice 18: 17-28.

40. Weidemann F, Herrmann S, Stork S, Markus Niemann, Stefan Frantz, et al. (2009) Impact of myocardial fibrosis in patients with symptomatic severe aortic stenosis. Circulation 120: 577-584.

41. Lancellotti P, Moonen M, Magne J, Kim O’Connor, Bernard Cosyns, et al. (2010) Prognostic effect of long-axis left ventricular dysfunction and B-type natriuretic peptide levels in asymptomatic aortic stenosis. Am J Cardiol 105: 383-388.

42. Kearney LG, Lu K, Ord M, S K Patel, K Profitis, et al. (2012) Global longitudinal strain is a strong independent predictor of all-cause mortality in patients with aortic stenosis. Eur Heart J Cardiovasc Imaging 13: 827-833.

43. Dahl JS, Videbaek L, Poulsen MK, Torsten R. Rudbæk, Patricia A. Pellikka, et al. (2012) Global strain in severe aortic valve stenosis: Relation to clinical outcome after aortic valve replacement. Circ Cardiovasc Imaging; 5: 613-620.

44. Voigt JU, Pedrizzetti G, Lysyansky P, et al. (2015) Definitions for a common standard for 2D speckle tracking echocardiography: consensus document of the EACVI/ASE/ Industry Task Force to standardize deformation imaging. Eur Heart J Cardiovasc Imaging 16: 1-11.

45. Cioffi G, Faggiano P, Vizzardi E, Luigi Tarantini, Dana Cramariuc, et al. (2011) Prognostic value of in appropriately high left ventricular mass in asymptomatic severe aortic stenosis. Heart 97: 301-317.

46. Julien Magne, Bernard Cosyns, Bogdan A. Helle G, Carstensen, Jordi Dahl, et al . (2019) Distribution and Prognostic Significance of Left Ventricular Global Longitudinal Strain in Asymptomatic Significant Aortic Stenosis: An Individual Participant Data Meta-Analysis. JACC: Cardiovascular Imaging 12: 84-92.

47. Elkilany, GEN, Ghobashi A, Salama M, Singh J (2020) Prevalence and Sub Clinical Detection of Concomitant Dilated Cardiomyopathy in Subjects with Bicuspid Aortic Valves. J Cardiol Cardiovasc Ther 16: 004-008.

48. Fernandes Jose M, Benício de Oliveira Romão, Ivan Romero Rivera, Maria Alayde Mendonça, Francisco de Assis Costa, et al. (2019) Clinical value of myocardial performance index in patients with isolated diastolic dysfunction; Cardiovascular Ultrasound 17: 1-9.

49. Redfield MM, Jacobsen SJ, Burnett JC Jr, Douglas W Mahoney, Kent Bailey, et al. (2003) Burden of systolic and diastolic ventricular dysfunction in the community: appreciating the scope of the heart failure epidemic. JAMA 289: 194-202.

50. Quinones MA, Gaasch WH, Alexander JK (1976) Influence of acute changes in preload, afterload, contractile state and heart rate on ejection and iso-volumic indices of myocardial contractility in man. Circulation 53: 293-302.

51. Liu D, Hu K, Herrmann S, Cikes M, Georg Ertl, et al. (2017) Value of tissue Doppler-derived Tei index and twodimensional speckle tracking imaging derived longitudinal strain on predicting outcome of patients with light-chain cardiac amyloidosis. Int J Cardiovasc Imaging 33: 837-845.

52. Meric M, Yesildag O, Yuksel S, Korhan Soylu, Muharrem Arslandag, et al. (2014) Tissue doppler myocardial performance index in patients with heart failure and its relationship with haemodynamic parameters. Int J Cardiovasc 
Imaging 30: 1057-1064.

53. Duzenli MA, Ozdemir K, Aygul N (2009) Comparison of myocardial performance index obtained either by conventional echocardiography or tissue Doppler echocardiography in healthy subjects and patients with heart failure. Heart Vessel 24: 8-15.

54. Johan A, Erik I, Ulf R, Bertil Andrén, Lars Lind (2004) Myocardial Performance Index, a Doppler-derived Index of Global Left Ventricular Function, Predicts Congestive Heart Failure in Elderly Men; Eur Heart J 25: 2220-2225.

55. Zoghbi WA, Adams D, Bonow RO, Maurice Enriquez-Sarano, Elyse Foster, et al. (2017) Recommendations for non-invasive evaluation of native valvular regurgitation: A report from the American Society of Echocardiography developed in collaboration with the Society for Cardiovascular Magnetic Resonance. J Am Soc Echocardiogr 30: 303-371.

56. Zilberszac R, Gabriel H, Schemper M, David Zahler, Martin Czerny, et al. (2013) Outcome of combined stenotic and regurgitant aortic valve disease. J Am Coll Cardiol; 61: 14891495..

57. Alashi A, Mentias A, Abdallah A, Ke Feng, A. Marc Gillinov, et al. (2018) Incremental Prognostic Utility of Left Ventricular Global Longitudinal Strain in Asymptomatic Patients With Significant Chronic Aortic Regurgitation and Preserved Left Ventricular Ejection Fraction. JACC Cardiovasc Imaging 11: 673-682.

58. Kusunose K, Agarwal S, Marwick TH, Brian P Griffin, Zoran B Popović (2014) Decision making in asymptomatic aortic regurgitation in the era of guidelines incremental values of resting and exercise cardiac dysfunction. Circ Cardiovasc Imaging 7: 352-362.

59. Alashi A, Khullar T, Mentias A, Douglas Johnston, A. Gillinov, et al. (2020) Long-Term Outcomes After Aortic Valve Surgery in Patients With Asymptomatic Chronic Aortic Regurgitation and Preserved LVEF: Impact of Baseline and Follow-Up Global Longitudinal Strain. JACC Cardiovasc Imaging 13: 10-1016.

60. Roushdy AM, Raafat SS, Shams KA, Maiy H El-Sayed (2016) Immediate and short-term effect of balloon mitral valvuloplasty on global and regional biventricular function : a two-dimensional strain echocardiographic study. Eur Heart J Cardiovasc Imaging 17: 316-325.

61. Prihadi EA, van der Bijl P, Dietz M, Rachid Abou, E Mara Vollema, et al. (2019) Prognostic Implications of Right Ventricular Free Wall Longitudinal Strain in Patients With Significant Functional Tricuspid Regurgitation; Circulation: Cardiovascular imaging 12: 1-9.

62. Chowdhury SM, Hijazi ZM, Fahey JT, John F Rhodes, Saibal Kar, et al. (2015) Speckle-Tracking Echocardiographic Measures of Right Ventricular Function Correlate With Improvement in Exercise Function After Percutaneous Pulmonary Valve Implantation. J Am Soc Echocardiogr 28: 1036-1044

63. Kim HM, Cho GY, Hwang IC, Hong-Mi Choi, Jun-Bean Park, et al. (2018) Myocardial Strain in Prediction of Outcomes After Surgery for Severe Mitral Regurgitation. JACC Cardiovasc Imaging 11: 1235-1244.

64. Galal Eldin Nagib-Elkilany, Ram B Singh, Krasimira Hristova, Dominik Pella, Mary Anne Sacayanan Olpindo, et al. (2019): Cardiovascular Imaging for Investigation of Cardiovascular Diseases. EC Cardiology 6: 163-166.

65. Hiemstra YL, Tomsic A, van Wijngaarden SE, Meindert Palmen, Robert J.M. Klautz, et al. (2020) Prognostic Value of Global Longitudinal Strain and Etiology After Surgery for Primary Mitral Regurgitation. JACC Cardiovasc Imaging

\section{3: $577-585$.}

66. Alashi A, Mentias A, Patel K, A Marc Gillinov, Joseph F Sabik, et al. (2016) Synergistic Utility of Brain Natriuretic Peptide and Left Ventricular Global Longitudinal Strain in Asymptomatic Patients With Significant Primary Mitral Regurgitation and Preserved Systolic Function Undergoing Mitral Valve Surgery; Circulation: Cardiovascular Imaging 9: $1-10$

67. Namazi F, van der Bijl P, Hirasawa K, Vasileios Kamperidis, Kensuke Hirasawa, et al. (2020) Prognostic Value of Left Ventricular Global Longitudinal Strain in Patients With Secondary Mitral Regurgitation. J Am Coll Cardiol 75: 750758.

68. Francesco G, Antonio R, Ferdinando P, Elena Biagini, Francesco Barberini, et al . (2015) Clinical Use of Doppler Echocardiography in Organic Mitral Regurgitation: From Diagnosis to Patients' Management. J Cardiovasc Ultrasound 23: 121-133.

69. Little SH, Chan KL, Burwash IG (2007) Impact of blood pressure on the Doppler echocardiographic assessment of severity of aortic stenosis. Heart 93: 848-855.

70. Rosenhek R, Zilberszac R, Schemper M, Martin Czerny, Gerald Mundigler, et al. (2010) Natural history of very severe aortic stenosis. Circulation 121: 151-156.

71. Henkel DM, Malouf JF, Connolly HM, Hector I. Michelena, Maurice E. Sarano, et al. (2012) Asymptomatic left ventricular systolic dysfunction in patients with severe aortic stenosis: Characteristics and outcomes. J Am Coll Cardiol 60: $2325-$ 2329.

72. Mare'chaux S, Hachicha Z, Bellouin A, Jean G Dumesnil, Patrick Meimoun, et al. (2010) Usefulness of exercise-stress echocardiography for risk stratification of true asymptomatic patients with aortic valve stenosis. Eur Heart J 31: 1390-1397.

73. Huded CP, Masri A, Kusunose K, Andrew L Goodman, Richard A Grimm, et al. (2018) Outcomes in Asymptomatic Severe Aortic Stenosis With Preserved Ejection Fraction Undergoing Rest and Treadmill Stress Echocardiography. J Am Heart Assoc 7: 1-20.

74. Dahl JS, Videbæk L, Poulsen MK, Torsten R. Rudbæk, Patricia A. Pellikka, et al. (2012) Global strain in severe aortic valve stenosis relation to clinical outcome after aortic valve replacement. Circ Cardiovasc Imaging 5: 613-620.

75. D'Andrea A, Carbone A, Agricola E, Lucia Riegler, Simona Sperlongano, et al. (2019) Predictive Value of Left Ventricular Myocardial Deformation for Left Ventricular Remodeling in Patients With Classical Low-Flow, Low-Gradient Aortic Stenosis Undergoing Trans catheter Aortic Valve Replacement. J Am Soc Echocardiogr 32: 730-736.

76. Magne J, Cosyns B, Popescu BA, Helle G. Carstensen, Jordi Dahl, et al. (2019) Distribution and Prognostic Significance of Left Ventricular Global Longitudinal Strain in Asymptomatic Significant Aortic Stenosis: An Individual Participant Data Meta-Analysis. JACC Cardiovasc Imaging 12: 84-92.

77. Dulgheru R, Pibarot P, Sengupta PP, Luc A Piérard, Raphael Rosenhek, et al. (2016) Multimodality Imaging Strategies for the Assessment of Aortic Stenosis: Viewpoint of the Heart Valve Clinic International Database (HAVEC) Group. Circ Cardiovasc Imaging 9: 1-14.

78. Dahl JS, Magne J, Pellikka PA, Donal E, Thomas H Marwick (2019) Marwick TH. Assessment of Subclinical Left Ventricular Dysfunction in Aortic Stenosis. JACC Cardiovasc Imaging 12: 163-171.

79. Yun-Hye Kim, Gum-Joo Choi, Chul Park (2018) Rate of left ventricular pressure change by Doppler echocardiography in dogs with chronic mitral valve disease at different stages of 
congestive heart failure; Vet Radiol Ultrasound 59: 758-766.

80. Cecconi M, De Backer D, Antonelli M, Richard Beale, Jan Bakker, et al. (2014) Consensus on circulatory shock and hemodynamic monitoring. Task force of the European Society of Intensive Care Medicine. Intensive Care Med 40: 179517815 .

81. Boissier F, Razazi K, Seemann A, Alexandre Bedet, Arnaud W Thille, et al. (2017) Left ventricular systolic dysfunction during septic shock: the role of loading conditions. Intensive Care Med 43: 633-642.

82. Geyer H, Caracciolo G, Abe H, Wilansky S, Carerj S, et al. (2010) Assessment of myocardial mechanics using speckle tracking echocardiography: fundamentals and clinical applications. J Am Soc Echocardiogr 23: 351-369..
83. Wallace AG, Skinner NS Jr, Mitchell JH (1963) Hemodynamic determinants of the maximal rate of rise of left ventricular pressure. Am J Phys 205: 30-36.

84. Suresh Chengode (2016) Left ventricular global systolic function assessment by echocardiography; Ann Card Anaesth 19: $26-34$

85. PETER E, JOERG G, ROSEMARIE K, J Carroll, H P Krayenbuehl (1982) Left Ventricular Relaxation in Patients with Left Ventricular Hypertrophy Secondary to Aortic Valve Disease; Circulation 65: 1395-1404.

86. RB Singh, Galal Najib Elkilany, Jan Fedacko, Krasimira Hristova (2016) Viewpoint: Utility and Necessity of Cardiovascular Imaging in a Chest Pain Unit; World Heart Journal 8: 125-131.
Copyright: (C)2020 Galaleldin Nagib Elkilany. This is an open-access article distributed under the terms of the Creative Commons Attribution License, which permits unrestricted use, distribution, and reproduction in any medium, provided the original author and source are credited. 